\title{
Article
}

\section{Stabilization and synchronization of hyper-chaotic financial system involved in positive interest rate}

\author{
Ruofeng Rao ${ }^{1,2}$ \\ 1 Department of Mathematics, Chengdu Normal University, Chengdu 61130, China; ruofengrao@163.com or \\ ruofengrao@cdnu.edu.cn \\ 2 Institute of Financial Mathematics, Chengdu Normal University, Chengdu 61130, China
}

\begin{abstract}
In real financial market, the delayed market feedback and the delayed effect of government macro-control are inevitable. And both the delay of market feedback and the delay of macro-control effect bring about the mathematical difficulties in studying stabilization and synchronization of the hyper-chaotic financial system. However, employing Lyapunov function method, differential mean value theorem, suitable bounded hypotheses and pulse control technology results in the globally asymptotical stabilization and synchronization criteria. It is the first paper to drive the stabilization and synchronization criteria under the assumptions of the double delays. Finally, numerical examples illuminate the effectiveness of the proposed methods.
\end{abstract}

Keywords: delayed feedback financial system; asymptotical stability; Lyapunov function; synchronization; impulse

\section{Introduction}

The complexity of economic systems often leads to unpredictable dynamic behavior. The periodic economic crisis reminds me that it is necessary to study and control the instability and chaos of financial system. Recently, many scholars have investigated the stability and synchronization of a class of chaotic finical system that is composed of the production sub-block, currency sub-block, securities sub-block, and labor sub-block (see, e.g. [1-9]). In many related literature([1-9]), the following finical system was investigated, which is composed of the production sub-block, currency sub-block, securities sub-block, and labor sub-block,

$$
\left\{\begin{array}{l}
\dot{x}=z+(y-a) x \\
\dot{y}=1-b y-x^{2} \\
\dot{z}=-x-c z,
\end{array}\right.
$$

where $x$ represents the interest rate, $y$ represents the investment demand, $z$ represents the price index, $a$ represents savings, $b$ represents the unit investment cost, and $c$ represents the elasticity of commodity demand.

Based on the improved financial chaos system model, the authors of [10] took the global economic crisis caused by American subprime mortgage crisis in 2007 as the background of the 
market model, adding a state variable (average profit rate) to the system (1.1), and proposed a new kind of hyper-chaotic financial system :

$$
\left\{\begin{array}{l}
\dot{x}=z+(y-a) x+u \\
\dot{y}=1-b y-x^{2} \\
\dot{z}=-x-c z \\
\dot{u}=-d x y-k u
\end{array}\right.
$$

which is hyper-chaotic. Indeed, set $a=0.8989, b=0.1989, c=1.499, d=0.2001, k=0.1699$, I can compute that there are three equilibrium points $P_{0}(0,5.0277,0,0), P_{1}(1.6590,-8.8101,-1.1067,17.2141)$ and $P_{2}(-1.6590,-8.8101,1.1067,17.2141)$ for the system (1.2). And three equilibrium points all are unstable saddle points.

On the other hand, both the market feedback delay and the delayed effect of the government's macroeconomic control are inevitable, but seldom papers involved in the above-mentioned multiple delays, which inspires me to write this paper. For the first time, the stabilization and synchronization are simultaneously considered for the hyper-chaotic financial system with the above double delays. And the newly-obtained results will provide some theoretical guidance for the actual financial market.

The rest of this paper is arranged as follows, in the second section, some system descriptions and preparations are presented. And the main results on stabilization and synchronization are given in the third section. Besides, in the fourth section, two numerical examples are proposed to illuminate the effectiveness of the new criteria. In final section, some conclusions are derived to elaborate further the main purpose and significance of this article.

\section{System descriptions and preparations}

From [10], there exist three equilibrium points for the system (1.2):

$$
P_{0}\left(0, \frac{1}{b}, 0,0\right), P_{1,2}\left( \pm \theta, \frac{k+a c k}{c(k-d)}, \mp \frac{\theta}{c}, \frac{d \theta(1+a c)}{c d-c k}\right) \text { with } \theta=\sqrt{\frac{k b+a b c k}{c(d-k)}+1} .
$$

Since the delayed feedback is a common phenomenon in the real market,I get the following delayed feedback model for the system (1.2),

$$
\left\{\begin{array}{l}
\dot{x}=z+(y-a) x+u+k_{1}\left(x-x\left(t-\tau_{1}(t)\right)\right) \\
\dot{y}=1-b y-x^{2}+k_{2}\left(y-y\left(t-\tau_{2}(t)\right)\right) \\
\dot{z}=-x-c z+k_{3}\left(z-z\left(t-\tau_{3}(t)\right)\right) \\
\dot{u}=-d x y-k u+k_{4}\left(u-u\left(t-\tau_{4}(t)\right)\right) .
\end{array}\right.
$$

Let $X=\left(x_{1}, x_{2}, x_{3}, x_{4}\right)^{T}$, and

$$
\left\{\begin{array}{l}
x_{1}=x-\theta \\
x_{2}=y-\frac{k+a c k}{c(k-d)} \\
x_{3}=z+\frac{\theta}{c} \\
x_{4}=u-\frac{d \theta(1+a c)}{c d-c k},
\end{array}\right.
$$


then the equilibrium point $P_{1}\left(\theta, \frac{k+a c k}{c(k-d)},-\frac{\theta}{c}, \frac{d \theta(1+a c)}{c d-c k}\right)$ with the positive interest rate $\theta>0$ of the system (1.2) corresponds to the zero solution of the following system

$$
\left\{\begin{array}{l}
\dot{x_{1}}=x_{3}-\frac{\theta}{c}+\left(x_{2}+\frac{k+a c k}{c(k-d)}-a\right)\left(x_{1}+\theta\right)+x_{4}+\frac{d \theta(1+a c)}{c d-c k}+k_{1}\left(x-x\left(t-\tau_{1}(t)\right)\right) \\
\dot{x_{2}}=1-b\left(x_{2}+\frac{k+a c k}{c(k-d)}\right)-\left(x_{1}+\theta\right)^{2}+k_{2}\left(y-y\left(t-\tau_{2}(t)\right)\right) \\
\dot{x_{3}}=-\left(x_{1}+\theta\right)-c\left(x_{3}-\frac{\theta}{c}\right)+k_{3}\left(z-z\left(t-\tau_{3}(t)\right)\right) \\
\dot{x_{4}}=-d\left(x_{1}+\theta\right)\left(x_{2}+\frac{k+a c k}{c(k-d)}\right)-k\left(x_{4}+\frac{d \theta(1+a c)}{c d-c k}\right)+k_{4}\left(u-u\left(t-\tau_{4}(t)\right)\right),
\end{array}\right.
$$

or

$$
\left\{\begin{aligned}
\dot{x_{1}} & =\left(\frac{k+a c k}{c(k-d)}-a\right) x_{1}+\theta x_{2}+x_{3}+x_{4}+x_{1} x_{2}+k_{1}\left(x-x\left(t-\tau_{1}(t)\right)\right) \\
\dot{x_{2}} & =-2 \theta x_{1}-b x_{2}-x_{1}^{2}+k_{2}\left(y-y\left(t-\tau_{2}(t)\right)\right) \\
\dot{x_{3}} & =-x_{1}-c x_{3}+k_{3}\left(z-z\left(t-\tau_{3}(t)\right)\right) \\
\dot{x_{4}} & =-\frac{d(k+a c k)}{c(k-d)} x_{1}-d \theta x_{2}-k x_{4}-d x_{1} x_{2}+k_{4}\left(u-u\left(t-\tau_{4}(t)\right)\right), \\
X(s) & =\phi(s), \quad s \in[-\tau, 0],
\end{aligned}\right.
$$

where $X=\left(x_{1}, x_{2}, x_{3}, x_{4}\right)^{T}$. Furthermore, the system (2.4) can be rewritten as the following system in form of vector-matrix,

$$
\left\{\begin{array}{l}
\dot{X}(t)=A X(t)+f(X(t))+K(X(t)-X(t-\tau(t))), \quad t \geqslant 0 \\
X(s)=\phi(s), \quad s \in[-\tau, 0]
\end{array}\right.
$$

where

$$
A=\left(\begin{array}{cccc}
\left(\frac{k+a c k}{c(k-d)}-a\right) & \theta & 1 & 1 \\
-2 \theta & -b & 0 & 0 \\
-1 & 0 & -c & 0 \\
-\frac{d(k+a c k)}{c(k-d)} & -d \theta & 0 & -k
\end{array}\right), \quad f(X)=\left(\begin{array}{c}
x_{1} x_{2} \\
-x_{1}^{2} \\
0 \\
-d x_{1} x_{2}
\end{array}\right), \quad K=\left(\begin{array}{cccc}
k_{1} & 0 & 0 & 0 \\
0 & k_{2} & 0 & 0 \\
0 & 0 & k_{3} & 0 \\
0 & 0 & 0 & k_{4}
\end{array}\right) .
$$

Remark 1. Obviously, $f(X)$ is not Lipschitz continuous.

Under the delayed impulse control on the system (2.5), I can get

$$
\left\{\begin{array}{l}
\dot{X}(t)=A X(t)+f(X(t))+K(X(t)-X(t-\tau(t))), \quad t \geqslant 0, t \neq t_{k} \\
X\left(t_{k}^{+}\right)-X\left(t_{k}^{-}\right)=D_{k} X\left(t_{k}-\rho_{k}\right), \quad k=1,2, \cdots \\
X(s)=\phi(s), \quad s \in[-\tau, 0]
\end{array}\right.
$$

or

$$
\left\{\begin{aligned}
\dot{x} & =z+(y-a) x+u+k_{1}\left(x-x\left(t-\tau_{1}(t)\right)\right), \quad t \geqslant 0, t \neq t_{k} \\
\dot{y} & =1-b y-x^{2}+k_{2}\left(y-y\left(t-\tau_{2}(t)\right)\right), \quad t \geqslant 0, t \neq t_{k} \\
\dot{z} & =-x-c z+k_{3}\left(z-z\left(t-\tau_{3}(t)\right)\right), \quad t \geqslant 0, t \neq t_{k} \\
\dot{u} & =-d x y-k u+k_{4}\left(u-u\left(t-\tau_{4}(t)\right)\right), \quad t \geqslant 0, t \neq t_{k}, \\
X\left(t_{k}^{+}\right) & -X\left(t_{k}^{-}\right)=D_{k} X\left(t_{k}-\rho_{k}\right), \quad k=1,2, \cdots \\
X(s) & =\phi(s), \quad s \in[-\tau, 0]
\end{aligned}\right.
$$


where $X$ is defined in (2.3). That is, the zero solution of the system of (2.7) corresponds to the equilibrium point $P_{1}\left(\theta, \frac{k+a c k}{c(k-d)},-\frac{\theta}{c}, \frac{d \theta(1+a c)}{c d-c k}\right)$ of the hyper-chaotic financial system (2.8).

Construct the following response system for the drive system (2.7),

$$
\left\{\begin{array}{l}
\dot{Y}(t)=A Y(t)+f(Y(t))+K(Y(t)-Y(t-\tau(t))), \quad t \geqslant 0, t \neq t_{k} \\
Y\left(t_{k}^{+}\right)-Y\left(t_{k}^{-}\right)=D_{k} Y\left(t_{k}-\rho_{k}\right), \quad k=1,2, \cdots \\
Y(s)=\psi(s), \quad s \in[-\tau, 0]
\end{array}\right.
$$

and the error system of pulse synchronization is given as follows,

$$
\left\{\begin{array}{l}
\dot{e}(t)=A e(t)+\breve{f}(e(t))+K(e(t)-e(t-\tau(t))), \quad t \geqslant 0, t \neq t_{k}, \\
e\left(t_{k}^{+}\right)-e\left(t_{k}^{-}\right)=D_{k} e\left(t_{k}-\rho_{k}\right), \quad k=1,2, \cdots \\
e(s)=\psi(s)-\phi(s), \quad s \in[-\tau, 0]
\end{array}\right.
$$

where $e=\left(e_{1}, e_{2}, e_{3}\right)^{T}=\left(Y_{1}-X_{1}, Y_{2}-X_{2}, Y_{3}-X_{3}\right)^{T}$ is the error of synchronization, and

$$
\breve{f}(e)=f(Y)-f(X)=\left(\begin{array}{c}
y_{1} y_{2}-x_{1} x_{2} \\
x_{1}^{2}-y_{1}^{2} \\
0 \\
d x_{1} x_{2}-d y_{1} y_{2}
\end{array}\right)
$$

Lemma 2.1([11, Theorem 3.2]). Under the assumptions of Theorem 3.1, the following fuzzy system (2.17) is bounded under the meaning of $L^{\infty}$ :

$$
\left\{\begin{array}{l}
\dot{X}(t)=\sum_{r=1}^{n} \varrho_{r}(\hat{\omega}(t)) H_{\sigma r} X(t)+f(X(t))-D_{\sigma} X(t)+\varphi_{\sigma}(X(t), X(t-\tau(t))) d w(t), \quad t \geqslant 0 \\
X(s)=\xi(s), \quad s \in[-\tau, 0] .
\end{array}\right.
$$

For convenience, I employ the following notations:

- For a symmetric matrix $A$, I denote by $\lambda_{\max } A$ the maximum eigenvalue of $A$;

- For a vector $v=\left(v_{1}, v_{2}, v_{3}\right)^{T} \in R^{3}$, I denote by $\|v\|=\sqrt{\sum_{i=1}^{3} v_{i}^{2}}$ the norm of $v$;

- For a matrix $A$, I denote by $\|A\|=\sqrt{\lambda_{\max }\left(A^{T} A\right)}$ the norm of $A$.

\section{Stabilization by impulse control}

At first, I may assume in this section that $X\left(t_{k}^{-}\right)=X\left(t_{k}\right)$ for all $k \in \mathbb{Z}^{+}$.

Secondly, from Lemma 2.1, I can similarly derive the boundedness of the system (2.7) and (2.8) under mild conditions. So in this paper, I may give the following boundedness assumptions:

(H1) Suppose that time delays $\tau_{i}(t) \in[0, \tau], i=1,2,3$. Assume that there are two positive scalars $M_{1}, M_{2}$ such that

$$
0<M_{1} \leqslant\|X(s)\|^{2} \leqslant M_{2}, \quad \forall s \in[-\tau,+\infty) .
$$

If the upper limit of delays $\tau$ is appropriately small, the following continuity hypothesis is natural due to the boundedness.

(H2) For any given $\tau>0$, there exists the corresponding positive number $c_{\tau}>0$ such that

$$
\left|\|X(t)\|^{2}-\|X(t-\tau(t))\|^{2}\right|<c_{\tau}
$$


In this section, I assume that time delays $\tau(t) \in[-\tau, 0]$, and $\rho_{k} \in[-\rho, 0]$ for all $k \in \mathbb{Z}^{+}$. In order to obtain the stability of the system, a certain pulse frequency is required, so I assume a smaller pulse interval as follows,

$$
\sup _{k \in \mathbb{Z}^{+}}\left(t_{k}-t_{k-1}\right)<c_{0}
$$

where $c_{0}$ is a positive number.

Mainly inspired by some methods and ideas of my another [20], I present the following Theorem.

Theorem 3.1. Suppose that the conditions $(H 1),(H 2)$ and (3.3) hold. And if

$$
\left[\left\|D_{k}+I\right\|+\rho_{k}\left\|D_{k}\right\| \cdot\left(\|A\|+\sqrt{M_{2}\left(1+d^{2}\right)}+2\|K\| \frac{\sqrt{M_{2}}}{\sqrt{M_{1}}}\right)\right] e^{\frac{c_{\lambda} c_{0}}{2}} \leqslant d_{0}<1, \quad \forall k \in \mathbb{Z}^{+},
$$

where $d_{0}$ is a positive scalar,

$$
c_{\lambda}=\lambda_{\max }\left[A+A^{T}+2 K+r_{1} I+\frac{1}{r_{1}} M_{2}\left(1+d^{2}\right) I+r_{2} K^{2}+\frac{1}{r_{2}}\left(1+\frac{c_{\tau}}{M_{1}}\right) I\right],
$$

and $I$ represents the identity matrix, $\rho_{k}<t_{k}-t_{k-1}$, for all $k \in \mathbb{Z}^{+}$, then the zero solution of the system (2.7) is globally asymptotically stable, and the equilibrium solution $P_{1}$ with the positive interest rate $\theta$ of the system (2.8) is globally asymptotically stable.

Proof. Consider the following Lyapunov function

$$
V(t)=\|X\|^{2}
$$

It follows from (H1) that

$$
\|f(X)\|^{2} \leqslant M_{2}\left(1+d^{2}\right)\|X\|^{2} .
$$

Next, (H1) and (H2) yield

$$
\begin{aligned}
& D^{+} V(t, X)=2 X^{T}[A X(t)+f(X(t))+K(X(t)-X(t-\tau(t)))] \\
\leqslant & \left.\left.X^{T}\left[A+A^{T}+2 K+r_{1} I+\frac{1}{r_{1}} M_{2}\left(1+d^{2}\right) I\right] X+\left.\left[r_{2}|X|^{T} K^{2}|X|+\frac{1}{r_{2}} \mid X(t-\tau(t))\right)\right|^{T} \mid X(t-\tau(t))\right) \mid\right] \\
\leqslant & \lambda_{\max }\left[A+A^{T}+2 K+r_{1} I+\frac{1}{r_{1}} M_{2}\left(1+d^{2}\right) I+r_{2} K^{2}+\frac{1}{r_{2}}\left(1+\frac{c_{\tau}}{M_{1}}\right) I\right] V(t, X), t \in\left(t_{k-1}, t_{k}\right], k \in \mathbb{Z}^{+} .
\end{aligned}
$$

Then I get

$$
\|X(t)\|^{2} \leqslant\left\|X\left(t_{k-1}^{+}\right)\right\|^{2} e^{c_{\lambda}\left(t-t_{k-1}\right)}, \quad t \in\left(t_{k-1}, t_{k}\right] .
$$

According to the impulsive condition, I have

$$
X\left(t_{k}^{+}\right)=X\left(t_{k}^{-}\right)+D_{k} X\left(t_{k}-\rho_{k}\right)=X\left(t_{k}\right)+D_{k} X\left(t_{k}-\rho_{k}\right)
$$

So I can conclude that there exists $\eta_{k i}(i=1,2,3)$ with $\eta_{k i} \in\left(t_{k}-\rho_{k}, t_{k}\right)$ such that

$$
\begin{aligned}
& \left.\left\|X\left(t_{k}^{+}\right)\right\|=\|\left(D_{k}+I\right) X\left(t_{k}\right)-\rho_{k} D_{k} \dot{X}\left(\eta_{k}\right)\right] \| \\
\leqslant & \left\|D_{k}+I\right\| \cdot\left\|X\left(t_{k}\right)\right\|+\rho_{k}\left\|D_{k}\right\| \cdot \| A X\left(\eta_{k}\right)+f\left(X\left(\eta_{k}\right)\right)+K\left(X\left(\eta_{k}\right)-X\left(\eta_{k}-\tau\left(\eta_{k}\right)\right) \|\right. \\
\leqslant & \left\|D_{k}+I\right\| \cdot\left\|X\left(t_{k}\right)\right\|+\rho_{k}\left\|D_{k}\right\|\left[\left(\|A\|+\sqrt{M_{2}\left(1+d^{2}\right)}\right)\left\|X\left(\eta_{k}\right)\right\|+\|K\| \cdot\left\|X\left(\eta_{k}\right)-X\left(\eta_{k}-\tau\left(\eta_{k}\right)\right)\right\|\right] \\
\leqslant & {\left[\left\|D_{k}+I\right\|+\rho_{k}\left\|D_{k}\right\| \cdot\left(\|A\|+\sqrt{M_{2}\left(1+d^{2}\right)}+\|K\| \frac{2 \sqrt{M_{2}}}{\sqrt{M_{1}}}\right)\right] e^{\frac{c_{1} c_{0}}{2}}\left\|X\left(t_{k-1}^{+}\right)\right\|, }
\end{aligned}
$$


which together with (3.4) implies that $\left\{\left\|X\left(t_{k}^{+}\right)\right\|\right\}_{k=1}^{\infty}$ is a convergent sequence with its limit being zero, where $X\left(\eta_{k}\right)=\left(x_{1}\left(\eta_{k 1}\right), x_{2}\left(\eta_{k 2}\right), x_{3}\left(\eta_{k 3}\right)\right)^{T}$. For any given $t \in\left(t_{k}, t_{k+1}\right]$, I get by (3.9)

$$
0 \leqslant\|X(t)\| \leqslant\left\|X\left(t_{k-1}^{+}\right)\right\| e^{\frac{c_{\lambda}}{2}\left(t-t_{k-1}\right)} \leqslant e^{\frac{c_{\lambda}}{2} c_{0}}\left\|X\left(t_{k-1}^{+}\right)\right\| \rightarrow 0, k \rightarrow \infty,
$$

which completes the proof.

Remark 2. The condition $\rho_{k}<t_{k}-t_{k-1}$ implies that every macro-control measure (pulse) of the government should be effective enough to see the pulse effect within each pulse interval. Besides, the condition $\sup _{k \in \mathbb{Z}^{+}}\left(t_{k}-t_{k-1}\right)<c_{0}$ guarantees pulse (Macro-control) of a certain frequency if $c_{0}>0$ is appropriate small. No matter how complex and chaos the financial system is, high-frequency active macro-control is conducive to the global asymptotical stability of the economic system.

To derive the synchronization criterion, I may consider the following boundedness assumptions:

(H3) There are two positive scalars $N_{1}, N_{2}$ such that

$$
0<N_{1} \leqslant\|e(s)\|^{2} \leqslant N_{2}, \quad \forall s \in[-\tau,+\infty)
$$

(H4) For any given $\tau>0$, there exists the corresponding positive number $d_{\tau}>0$ such that

$$
\left|\|e(t)\|^{2}-\|e(t-\tau(t))\|^{2}\right|<d_{\tau}
$$

Theorem 3.2. Assume that $X, Y$ satisfy the boundedness conditions (H1) and (H2), and the error variable $e$ satisfies the boundedness assumptions (H3)-(H4). If, in addition, the following condition holds,

$$
\left[\left\|D_{k}+I\right\|+\rho_{k}\left\|D_{k}\right\| \cdot\left(\|A\|+\sqrt{4 M_{2}\left(2+d^{2}\right)}+\|K\| \frac{2 \sqrt{N_{2}}}{\sqrt{N_{1}}}\right)\right] e^{\frac{d_{\lambda} c_{0}}{2}} \leqslant d_{0}<1,
$$

then the system (2.9) can be globally exponentially synchronized onto the system (2.7), where

$$
d_{\lambda}=\lambda_{\max }\left[A+A^{T}+2 K+r_{1} I+\frac{4}{r_{1}} N_{2}\left(2+d^{2}\right) I+r_{2} K^{2}+\frac{1}{r_{2}}\left(1+\frac{d_{\tau}}{N_{1}}\right) I\right] .
$$

Proof. Consider the following Lyapunov function

$$
V(t)=\|e\|^{2} .
$$

Similarly, I can conclude from the assumptions of Theorem 3.2 that

$$
\begin{aligned}
\|\breve{f}(e)\|^{2} & \leqslant 2\left(1+d^{2}\right)\left[\|X\|^{2}+\|Y\|^{2}\right]\|e\|^{2}+2\left(\|X\|^{2}+\|Y\|^{2}\right)\|e\|^{2} \\
& \leqslant 4\left(1+d^{2}\right) M_{2}\|e\|^{2}+4 M_{2}\|e\|^{2}=4\left(2+d^{2}\right) M_{2}\|e\|^{2},
\end{aligned}
$$

and

$$
\begin{aligned}
& D^{+} V(t, e)=2 e^{T}(t)[A e(t)+\breve{f}(e(t))+K(e(t)-e(t-\tau(t)))] \\
\leqslant & e^{T}\left[A+A^{T}+2 K+r_{1} I+\frac{4}{r_{1}} N_{2}\left(2+d^{2}\right) I+r_{2} K^{2}+\frac{1}{r_{2}}\left(1+\frac{d_{\tau}}{N_{1}}\right) I\right] e \\
\leqslant & \lambda_{\max }\left[A+A^{T}+2 K+r_{1} I+\frac{4}{r_{1}} N_{2}\left(2+d^{2}\right) I+r_{2} K^{2}+\frac{1}{r_{2}}\left(1+\frac{d_{\tau}}{N_{1}}\right) I\right] V(t, e), t \in\left(t_{k-1}, t_{k}\right], k \in \mathbb{Z}^{+} .
\end{aligned}
$$

Then I get

$$
\|e(t)\|^{2} \leqslant\left\|e\left(t_{k-1}^{+}\right)\right\|^{2} e^{d_{\lambda}\left(t-t_{k-1}\right)} \Rightarrow\|X(t)\| \leqslant\left\|X\left(t_{k-1}^{+}\right)\right\| e^{\frac{d_{\lambda}}{2}\left(t-t_{k-1}\right)}, \quad t \in\left(t_{k-1}, t_{k}\right] .
$$


According to the impulsive condition, I have

$$
e\left(t_{k}^{+}\right)=e\left(t_{k}^{-}\right)+D_{k} e\left(t_{k}-\rho_{k}\right)=e\left(t_{k}\right)+D_{k} e\left(t_{k}-\rho_{k}\right)
$$

So I deduce that there exists $\eta_{k i}(i=1,2,3)$ with $\eta_{k i} \in\left(t_{k}-\rho_{k}, t_{k}\right)$ such that

$$
\begin{aligned}
& \left\|e\left(t_{k}^{+}\right)\right\| \leqslant\left\|D_{k}+I\right\| \cdot\left\|e\left(t_{k}\right)\right\|+\rho_{k}\left\|D_{k}\right\| \cdot \| A e\left(\eta_{k}\right)+\breve{f}\left(e\left(\eta_{k}\right)\right)+K\left(e\left(\eta_{k}\right)-e\left(\eta_{k}-\tau\left(\eta_{k}\right)\right) \|\right. \\
\leqslant & \left\|D_{k}+I\right\| \cdot\left\|e\left(t_{k}\right)\right\|+\rho_{k}\left\|D_{k}\right\|\left[\left(\|A\|+\sqrt{4 M_{2}\left(2+d^{2}\right)}\right)\left\|e\left(\eta_{k}\right)\right\|+\|K\| \cdot\left\|e\left(\eta_{k}\right)-e\left(\eta_{k}-\tau\left(\eta_{k}\right)\right)\right\|\right] \\
\leqslant & \left\|D_{k}+I\right\| \cdot\left\|e\left(t_{k-1}^{+}\right)\right\| e^{\frac{d_{\lambda}}{2}\left(t-t_{k-1}\right)}+\rho_{k}\left\|D_{k}\right\|\left(\|A\|+\sqrt{4 M_{2}\left(2+d^{2}\right)}+\|K\| \frac{2 \sqrt{N_{2}}}{\sqrt{N_{1}}}\right)\left\|e\left(t_{k-1}^{+}\right)\right\| e^{\frac{d_{\lambda}}{2}\left(t-t_{k-1}\right)} \\
\leqslant & {\left[\left\|D_{k}+I\right\|+\rho_{k}\left\|D_{k}\right\| \cdot\left(\|A\|+\sqrt{4 M_{2}\left(2+d^{2}\right)}+\|K\| \frac{2 \sqrt{N_{2}}}{\sqrt{N_{1}}}\right)\right] e^{\frac{d_{\lambda} c_{0}}{2}}\left\|e\left(t_{k-1}^{+}\right)\right\|, }
\end{aligned}
$$

which together with (3.10) implies that $\left\{\left\|e\left(t_{k}^{+}\right)\right\|\right\}_{k=1}^{\infty}$ is a convergent sequence with its limit being zero, where $e\left(\eta_{k}\right)=\left(x_{1}\left(\eta_{k 1}\right), x_{2}\left(\eta_{k 2}\right), x_{3}\left(\eta_{k 3}\right)\right)^{T}$. For any given $t \in\left(t_{k}, t_{k+1}\right]$, I get by (3.15)

$$
0 \leqslant\|e(t)\| \leqslant\left\|e\left(t_{k-1}^{+}\right)\right\| e^{\frac{d_{\lambda}}{2}\left(t-t_{k-1}\right)} \leqslant e^{\frac{d_{\lambda}}{2} c_{0}}\left\|e\left(t_{k-1}^{+}\right)\right\| \rightarrow 0, k \rightarrow \infty,
$$

which completes the proof.

Remark 3. It is the first paper to derive the synchronization criterion for the systems (2.9) and (2.7) under the assumptions of the double delays.

\section{Numerical examples}

Example 4.1. Consider the system (2.7) or (2.8) with the following data:

$$
\begin{gathered}
M_{1}=1, M_{2}=8, \tau=0.5, c_{\tau}=0.3, \rho_{k} \equiv 0.01, D_{k} \equiv-\frac{4}{5} I, r_{1}=r_{2}=r_{3}=r_{4}=1, \\
K=\left(\begin{array}{cccc}
0.05 & 0 & 0 & 0 \\
0 & 0.07 & 0 & 0 \\
0 & 0 & 0.03 & 0 \\
0 & 0 & 0 & 0.033
\end{array}\right)
\end{gathered}
$$

Below I may consider two different data for comparisons:

\section{Case 1.}

$$
a=0.3, b=0.2, c=0.5, d=0.11, k=0.81, c_{0}=0.15,
$$

then direct computation yields that $\theta=\sqrt{\frac{k b+a b c k}{c(d-k)}+1}=0.6839$,

$$
A=\left(\begin{array}{cccc}
2.3614 & 0.6839 & 1.0000 & 1.0000 \\
-1.3678 & -0.2000 & 0 & 0 \\
-1.0000 & 0 & -0.5000 & 0 \\
-0.2928 & -0.0752 & 0 & -0.8100
\end{array}\right)
$$

Further computation derives that $\|A\|=3.2952,\|K\|=0.07, c_{\lambda}=18.0898$. Let $d_{0}=0.99$ and direct computation results in

$$
\left[\left\|D_{k}+I\right\|+\rho_{k}\left\|D_{k}\right\| \cdot\left(\|A\|+\sqrt{M_{2}\left(1+d^{2}\right)}+2\|K\| \frac{\sqrt{M_{2}}}{\sqrt{M_{1}}}\right)\right] e^{\frac{c_{\lambda} c_{0}}{2}}=0.8021 \leqslant 0.99=d_{0}<1, \quad \forall k \in \mathbb{Z}^{+}
$$


which implies that the condition (3.4) is satisfied, and the equilibrium solution $P_{1}$ with the positive interest rate $\theta=68.39 \%$ of the system (2.8) is globally asymptotically stable due to Theorem 3.1.

Case 2.

$$
a=0.9, b=0.2, c=0.5, d=0.11, k=0.81, c_{0}=0.17,
$$

then direct computation yields that $\theta=\sqrt{\frac{k b+a b c k}{c(d-k)}+1}=0.5735$,

$$
A=\left(\begin{array}{cccc}
2.4557 & 0.5735 & 1.0000 & 1.0000 \\
-1.1469 & -0.2000 & 0 & 0 \\
-1.0000 & 0 & -0.5000 & 0 \\
-0.3691 & -0.0631 & 0 & -0.8100
\end{array}\right)
$$

Further computation derives that $\|A\|=3.2922,\|K\|=0.07, c_{\lambda}=18.2325$. Let $d_{0}=0.99$ and direct computation results in

$$
\left[\left\|D_{k}+I\right\|+\rho_{k}\left\|D_{k}\right\| \cdot\left(\|A\|+\sqrt{M_{2}\left(1+d^{2}\right)}+2\|K\| \frac{\sqrt{M_{2}}}{\sqrt{M_{1}}}\right)\right] e^{\frac{c_{\lambda} c_{0}}{2}}=0.9729 \leqslant 0.9=d_{0}<1, \quad \forall k \in \mathbb{Z}^{+},
$$

which implies that the condition (3.4) is satisfied, and the equilibrium solution $P_{1}$ with the positive interest rate $\theta=57.35 \%$ of the system (2.8) is globally asymptotically stable due to Theorem 3.1.

Table 1.Comparisons of positive interest rate and pulse interval

\begin{tabular}{|l|c|c|}
\hline & interest rate $\theta$ & pulse interval $c_{0}$ \\
\hline Case 1 & $68.39 \%$ & 0.15 \\
\hline Case 2 & $57.35 \%$ & 0.17 \\
\hline
\end{tabular}

Remark 4. Table 1 illuminates that when the system reach stable, the higher the interest rate, the smaller the pulse interval. This shows, in order to reach a balance of higher interest rates in the financial market, the government should speed up the pace of macro-control of the economy.

Example 4.2.Consider the following data for the systems (2.7),(2.9) and (2.10):

$$
\begin{gathered}
N_{1}=0.1, N_{2}=0.5, M_{1}=1, M_{2}=8, \tau=0.5, c_{\tau}=0.3, \rho_{k} \equiv 0.001, D_{k} \equiv-\frac{4}{5} I, r_{1}=r_{2}=r_{3}=r_{4}=1, \\
a=0.9, b=0.2, c=0.5, d=0.11, k=0.81, c_{0}=0.01 \\
K=\left(\begin{array}{cccc}
0.05 & 0 & 0 & 0 \\
0 & 0.07 & 0 & 0 \\
0 & 0 & 0.03 & 0 \\
0 & 0 & 0 & 0.033
\end{array}\right) .
\end{gathered}
$$

and then

$$
A=\left(\begin{array}{cccc}
2.3614 & 0.6839 & 1.0000 & 1.0000 \\
-1.3678 & -0.2000 & 0 & 0 \\
-1.0000 & 0 & -0.5000 & 0 \\
-0.2928 & -0.0752 & 0 & -0.8100
\end{array}\right)
$$

Further computations derive

$$
\left[\left\|D_{k}+I\right\|+\rho_{k}\left\|D_{k}\right\| \cdot\left(\|A\|+\sqrt{4 M_{2}\left(2+d^{2}\right)}+\|K\| \frac{2 \sqrt{N_{2}}}{\sqrt{N_{1}}}\right)\right] e^{\frac{d_{\lambda} c_{0}}{2}}=0.9055 \leqslant 0.99=d_{0}<1,
$$

which implies that the condition (3.10) holds. And hence, Theorem 3.2 tells me that the system (2.9) can be globally exponentially synchronized onto the system (2.7). 
Remark 5. Example 4.2 shows the feasibility of Theorem 3.2.

\section{Conclusions}

Interest rates are always positive at most of countries in the world when the economies are in balance. So my Theorem 3.1 and Theorem 3.2 illustrate theoretical guidance significance for the actual financial market. In particular, Theorem 3.1 shows that positive and correct macroeconomic control measures with a certain frequency are conducive to market balance and high positive interest rates. Finally, two numerical examples shows the effectiveness and feasibility of stabilization and synchronization criteria.

Funding: The work is supported by the Application basic research project of science and Technology Department of Sichuan Province (No. 2020YJ0434) and the Major scientific research projects of Chengdu Normal University in 2019 (No. CS19ZDZ01).

Conflicts of Interest: The author declares no conflict of interest.

\section{References}

1. Cheng, S. Complicated Science and Management. Article Collection of Beijing Xiangshan Conference; Science Press: Beijing, China, 1998; pp. 1-9. (In Chinese)

2. Huang, D.; Li, H. Theory and Method of Nonlinear Economics; Sichuan University Press: Chengdu, China, 1993.

3. Ma, J.; Chen, Y. Study for the bifurcation topological structure and the global complicated character of a kind of nonlinear finance system (I). Appl. Math. Mech. 2001, 11, 1240-1251.

4. Ma, J.; Chen, Y. Study for the bifurcation topological structure and the global complicated character of a kind of nonlinear finance system (II). Appl. Math. Mech. 2001, 12, 1375-1382.

5. Chen, W. Dynamics and control of a financial system with time-delayed feedbacks. Chaos Solitons Fractals 2008, 37, 1198-1207.

6. Zhao, M.; Wang, J. $H_{\infty}$ control of a chaotic finance system in the presence of external disturbance and input time-delay. Appl. Math. Comput. 2014, 233, 320-327.

7. Zhao, X.; Li, Z.; Li, S. Synchronization of a chaotic finance system. Appl. Math. Comput. 2011, 217, 6031-6039

8. Ruofeng Rao. Global Stability of a Markovian Jumping Chaotic Financial System with Partially Unknown Transition Rates under Impulsive Control Involved in the Positive Interest Rate. Mathematics, 2019, 7(7), 579

9. Ruofeng Rao and Shouming Zhong. Impulsive control on delayed feedback chaotic financial system with Markovian jumping. Advances in Difference Equations, 2020, 2020:50

10. Yu H J, Cai G L, Li Y X. Dynamic analysis and control of a new hyperchaotic finance system. Nonlinear Dynamics. 2012, 67(3): 2171-2182.

11. Zhilin Pu, Ruofeng Rao, Shouming Zhong. Switching design of Macro-controlling intermittently on chaotic stochastic financial system with delayed feedback under fuzzy estimating condition.

12. Hui Wang, Quanxin Zhu. Adaptive output feedback control of stochastic nonholonomic systems with nonlinear parameterization. Automatica, 2018,98, 247-255

13. Xiaodi Li, Xueyan Yang, Jinde Cao. Event-triggered impulsive control for nonlinear delay systems Automatica, 2020, 117, 108981

14. Quanxin Zhu. Stabilization of Stochastic Nonlinear Delay Systems With Exogenous Disturbances and the Event-Triggered Feedback Control. IEEE Transactions on Automatic Control, 2019, 64(9).

15. Xiaodi Li, Xueyan Yang. Lyapunov stability analysis for nonlinear systems with state-dependent state delay. Automatica, 2020, 112, 108674

16. Quanxin Zhu. Stability analysis of stochastic delay differential equations with Levy noise Systems \& Control Letters, 2018, 118, 62-68

17. Quanxin Zhu, Hui Wang. Output feedback stabilization of stochastic feedforward systems with unknown control coefficients and unknown output function. Automatica, 2018, 87, 166-175

18. Xiaodi Li, Xueyan Yang, Shiji Song. Lyapunov conditions for finite-time stability of time-varying time-delay systems. Automatica, 2019, 103, 135-140

19. Wei Hu, Quanxin Zhu, Hamid Reza Karimi. Some Improved Razumikhin Stability Criteria for Impulsive Stochastic Delay Differential Systems. IEEE Transactions on Automatic Control, 2019, 64(12). 
10 of 10

20. Ruofeng Rao, Shouming Zhong. Input-to-state stability and no-inputs stabilization of delayed feedback chaotic financial system involved in open and closed economy. Discrete \& Continuous Dynamical Systems, Ser. S, doi: 10.3934 /dcdss. 2020280 